Article

\title{
Capacity of the Weakly Absorbent Turbulent Ocean Channel with the Coaxial Double-Position Power Gaussian Vortex
}

\author{
Qingze Yan, Yun Zhu and Yixin Zhang *
}

School of Science, Jiangnan University, Wuxi 214122, China; Yanqz0620@163.com (Q.Y.); zhuyun1210@163.com (Y.Z.)

* Correspondence: zyx@jiangnan.edu.cn

check for updates

Citation: Yan, Q.; Zhu, Y.; Zhang, Y. Capacity of the Weakly Absorbent Turbulent Ocean Channel with the Coaxial Double-Position Power Gaussian Vortex. J. Mar. Sci. Eng. 2021, 9, 1117. https://doi.org/ 10.3390/jmse9101117

Academic Editor: Nikitas Nikitakos

Received: 30 August 2021

Accepted: 1 October 2021

Published: 14 October 2021

Publisher's Note: MDPI stays neutral with regard to jurisdictional claims in published maps and institutional affiliations.

Copyright: (C) 2021 by the authors. Licensee MDPI, Basel, Switzerland. This article is an open access article distributed under the terms and conditions of the Creative Commons Attribution (CC BY) license (https:/ / creativecommons.org/licenses/by/ $4.0 /)$.
Abstract: Turbulence and absorption of seawater are two important factors affecting the signal transmission quality of underwater optical communication link. Here, we study the channel capacity and bit error rate of an underwater extinction communication link with a coaxial double-position power Gaussian vortex carrier based on Rytov approximation theory. The study finds that channel capacity and bit error rate are the nonlinear functions of the dimensionless structural parameter and reach maximum and minimum values at $|\alpha|=1$, respectively. The seawater absorption has a great influence on the channel capacity but not bit error rate. The communication link with large receiving aperture, small transmitting beam diameter, long wavelength of light source in a seawater window, and more OAM channels has high channel capacity.

Keywords: oceanic turbulence; seawater absorption; position power Gaussian vortex; channel capacity

\section{Introduction}

Orbital angular momentum (OAM) is a new dimension that can be encoded, which can be adopted by optical [1-4] and acoustic [5-7] communication systems to improve the information capacity of links. Although acoustic OAM has the advantages of low loss of underwater communication and perfect theoretical [5-7] and experimental model compared with underwater optical OAM transmission, its fatal weakness is the low channel capacity and signal transmission rate of underwater acoustic communication link. Therefore, as underwater communication and detection technology requires higher communication link channel capacity and signal transmission rate, OAM optical communication has become one of the new focuses in the field of underwater communication.

However, due to the presence of various constituents in the ocean and oceanic turbulence, seawater scattering and absorption inevitably limit the transmission of information carried by OAM modes over optical communication links [8-18]. Therefore, in order to reduce the above negative effects of seawater and consider the relationship between signal transmission probability and channel capacity, a lot of studies have been conducted from the perspectives of OAM detection probability and link channel capacity. The disturbance degree of ocean turbulence to the detection probability of OAM mode of partially coherent Laguerre-Gaussian beams increases with the increase in beam radial mode order, OAM mode topological charge and ocean turbulence strength, and fully coherent vortex beams provided better performance than partially coherent ones [8]. The partially coherent elegant Laguerre-Gaussian beams are also more affected by turbulence as compared to the fully coherent elegant Laguerre-Gaussian beams, and one can choose optimum beam source to mitigate the influence of oceanic turbulence [9]. Although the detection probability of fractional OAM mode is lower than that of adjacent integer OAM mode, the channel capacity of the link can be increased by using fractional OAM mode when the available OAM topological charges are limited [10]. Compared with Laguerre-Gaussian beams, the Bessel Gaussian beam transmitted in turbulent seawater has stronger anti-turbulence and better OAM transmission performance [11]. For partially coherent modified Bessel 
correlated vortex beam, the effect of high-anisotropic ocean turbulence on OAM signal is weaker than that of low-anisotropic ocean turbulence. In addition, the mode with low OAM quantum number can be selected to improve the information transmission of the link, and the OAM mode with large energy level difference between neighboring OAM can be used to reduce the impact of turbulence, to improve the information capacity of the optical communication link [12]. The channel capacity of the link increases as the pulse width of the half-modulated input pulse increases and the Bessel cone angle decreases. Channel capacity is more sensitive to salinity fluctuations than temperature fluctuations. In anisotropic and isotropic turbulence, the internal and external scales of turbulence have different effects on channel capacity [13]. An airy-OAM beam with a smaller topological charge, larger main ring radius, and longer wavelength has larger detection probability [14]. The Lommel Gaussian beam, which has a small asymmetric coefficient, low OAM quantum number, optimal waist width, and long transmission window wavelength, has a low probability of crosstalk introduced by turbulence. [15]. The self-focusing characteristic of a perfect optical vortex beam is beneficial to the propagation of the OAM mode, and the perfect optical vortex beam with smaller topological charge, smaller initial radius, and optimized half-ring width can reduce the degradation effect of the PV beam caused by turbulence [16]. The normalized received probability of signal OAM modes carried by finite energy frozen wave is independent of the quantum number of OAM modes in weak seawater turbulence, and the higher normalized received probability achieved in the smaller received diameter of the receiver, the larger the transverse size and longer the signal wavelength and the signal OAM modes [17]. An HyGG vortex beam with smaller topological charge and longer wavelength has higher transmission quality [18]. Compared with the Laguerre-Gaussian beam and the Bessel-Gaussian beam, a diffraction- and attenuation-resistant random vortex beam is a more resistant to seawater turbulence and seawater absorption and the optical communication link, which consists of a long-wave length anti-diffraction and anti-attenuation random vortex carrier and a small receiving diameter, has high channel capacity [19]. More importantly, by designing a longitudinal profile of finite energy frozen beams, we can compensate the attenuation loss of the transmittance [20]. Moreover, the localized wave of Bessel-Gaussian amplitude envelope (or Bessel-Gaussian localized vortex) is a more suitable beam for the vortex mode communication than conventional vortex waves [21,22]. The disturbance of turbulent seawater to the OAM modes of anti-diffraction and anti-attenuation random beam with a large truncated Gaussian aperture is less than the corresponding OAM modes carried by the Laguerre-Gaussian beam [23].

The position-power Gaussian vortex is a new kind of vortex beam, which is different from all the previous research. The disturbance of seawater turbulence when the OAM mode carried by the beam is transmitted in the underwater channel is also a problem worth discussing. Furthermore, most of these studies only focus on the transport of a single OAM vortex or multiple OAM vortices with the same rotation direction in turbulent seawater. When the vortex carrier carries two OAM modes with different amplitudes and rotation directions in the absorption of turbulent seawater, whether the transmission characteristics are different from the above research conclusions, that is, whether there are more factors that can optimize the communication system. However, as far as we know, there are no reports on the channel capacity of underwater communication links with a coaxial dual-position power vortex Gaussian carrier.

In this paper, the influence of coaxial double-position power vortex Gaussian carrier parameters and seawater turbulence parameters on the channel capacity and bit error rate (BER) of the communication link is studied. The remainder of this paper is organized as follows. In Section 2, we derive the random coaxial double-position power Gaussian vortex (RCDPPGV) laser based on the Huygens-Fresnel diffraction integral formula and the Rytov approximation theory. We derive the BER of OAM mode and the channel capacity of the optical communication link with a RCDPPGV carrier in Section 3. In Section 4, we discuss 
the effects of the parameters of turbulent absorption seawater and beam structure on the channel capacity as well as BET. Section 5 provides the main conclusions of the paper.

\section{Kolmogorov Ocean Spectrum and Random Coaxial Double-Position Power Gaussian Vortex}

This section mainly discusses two parts of your content. First, we briefly discuss the seawater spectrum. Secondly, the random coaxial double-position power Gaussian vortex is established by Rytov theory.

\subsection{Kolmogorov Ocean Spectrum}

For a liner, isotropic, homogeneous, and clean absorbing seawater, the refractive index of seawater is [19-21]

$$
n_{o c}=n_{r}+i n_{i}
$$

where $n_{r}$ is the mean value of real part of refractive index of seawater, $n_{i}$ is the mean value of imaginary part of refractive index of seawater that depends on the type of seawater, and $i=\sqrt{-1}$ is the imaginary symbol.

The turbulent characteristics of seawater are described by the following turbulent spectrum equation [24]

$$
\begin{aligned}
& \Phi_{n}(\kappa)=0.267 \times 10^{-8 \frac{\varepsilon^{-1 / 3}}{\chi_{T} \kappa^{-11 / 3}}} \omega^{2}\left[1+4.6\left(\kappa l_{0}\right)^{2 / 3}\right]\left[1-\exp \left(-\frac{\kappa^{2}}{\kappa_{0}^{2}}\right)\right] \\
& \times\left[\omega^{2} \exp \left(-\frac{\kappa^{2}}{\kappa_{T}^{2}}\right)+1 \exp \left(-\frac{\kappa^{2}}{\kappa_{S}^{2}}\right)-2 \omega \exp \left(-\frac{\kappa^{2}}{\kappa_{T S}^{2}}\right)\right] 0<\kappa<\infty,
\end{aligned}
$$

where $\kappa$ is the spatial frequency of turbulent fluctuations, $\varepsilon$ is the rate of dissipation of kinetic energy per unit mass of fluid ranging from $10^{-10} \mathrm{~m}^{2} / \mathrm{s}^{3}$ to $10^{-1} \mathrm{~m}^{2} / \mathrm{s}^{3}, \chi_{T}$ is the dissipation rate of the mean-squared temperature and has the range from $10^{-10} \mathrm{~K}^{2} / \mathrm{s}$ to $10^{-2} \mathrm{~K}^{2} / \mathrm{s}, l_{0}$ is the inner scale, $\kappa_{0}=2 \pi / L_{0}, L_{0}$ is the outer scale of turbulence, $\kappa_{T}=R_{T} / l$, $\kappa_{S}=R_{S} / l_{0}$, and $\kappa_{T S}=R_{T S} / l_{0} . R_{j}=\sqrt{3}\left[W_{j}-1 / 3+1 /\left(9 W_{j}\right)\right]^{3 / 2} Q^{-3 / 2},(j=T, S, T S)$, $W_{j}=\left\{\left[\frac{\operatorname{Pr}_{j}^{2}}{\left(6 \beta Q^{-2}\right)^{2}}-\frac{\operatorname{Pr}_{j}}{81 \beta Q^{-2}}\right]^{1 / 2}-\left[\frac{1}{27}-\frac{\operatorname{Pr}_{j}}{6 \beta Q^{-2}}\right]\right\}^{1 / 3}, Q=2.35, \operatorname{Pr}_{T}$ and $\operatorname{Pr}_{S}$, respectively, represent the Prandtl number of the temperature and salinity, $\operatorname{Pr}_{T S}=2 \operatorname{Pr}_{T} \operatorname{Pr}_{S} /\left(\operatorname{Pr}_{T}+\operatorname{Pr}_{S}\right), \omega$ defines the ratio of temperature and salinity contributions to the refractive index spectrum, which can vary in the interval $[-5,0]$ in the seawaters, $\omega=-5$ temperature is dominant in the induction of optical turbulence, while $\omega=0$ salinity is dominant in the induction of optical turbulence.

Compared with the atmospheric turbulence spectrum [24], Equation (2) can be rewritten into the following ocean spectrum

$$
\Phi_{n}(\kappa)=\varphi_{n}(\kappa) f(\omega, \kappa, \eta), \quad 0<\kappa<\infty,
$$

with "Kolmogorov ocean spectrum"

$$
\varphi_{n}(\kappa)=0.033 C_{\varepsilon \chi_{T}}^{2} \kappa^{-11 / 3}, 1 / \eta<\kappa<1 / L_{0},
$$

Additionally,

$$
\begin{aligned}
f(\omega, \kappa, \eta) & =\left[1+4.6\left(\kappa l_{0}\right)^{2 / 3}\right](1-\omega)^{-2}\left[1-\exp \left(-\frac{\kappa^{2}}{\kappa_{0}^{2}}\right)\right] \\
& \times\left[\exp \left(-\frac{\kappa^{2}}{\kappa_{T}^{2}}\right)+\frac{1}{\omega^{2}} \exp \left(-\frac{\kappa^{2}}{\kappa_{S}^{2}}\right)-\frac{2}{\omega} \exp \left(-\frac{\kappa^{2}}{\kappa_{T S}^{2}}\right)\right] .
\end{aligned}
$$

Here, $C_{\varepsilon \chi_{T}}^{2}=0.809 \times 10^{-7} \varepsilon^{-1 / 3} \chi_{T} \omega^{-2}(1-\omega)^{2}$ defines the structural constants of the salinity-temperature fluctuation of oceanic turbulence. 
Consider that the physical mechanism behind the oceanic turbulence and atmospheric turbulence are same and in conjunction with the above discussion, using Equation (5), we can just write Rytov variance for a plane wave in a homogeneous isotropic weakly turbulent ocean as

$$
\sigma_{1 \varepsilon \chi_{T}}^{2}=1.23 C_{\varepsilon \chi_{T}}^{2} k^{7 / 6} z^{11 / 6}
$$

Formally, Equation (6) is the same as the Rytov variance of plane waves in the turbulent atmosphere, except that $C_{\varepsilon X_{T}}^{2}$ is replaced by the structure constant of atmospheric turbulence $\mathrm{C}_{n}^{2}$. Of course, from the turbulence effect theory of light transmission in the atmosphere [25], we can directly obtain the conditions that seawater is in a weak fluctuation state, that is, $\sigma_{1 \varepsilon \chi_{T}}^{2}<1$.

\subsection{Random Coaxial Double-Position Power Gaussian Vortex}

In Figure 1, we give a schematic diagram of the rectangular coordinates $\left(x^{\prime}, y^{\prime}, z^{\prime}\right)$ and the communication link for signal transmission from the light source plane $\left(x^{\prime}, y^{\prime}, z^{\prime}\right)$ to the receiving plane $(x, y, z)$.

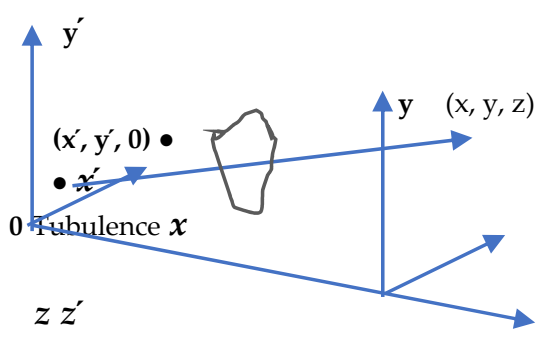

Figure 1. The schematic diagram of a signal transmission from the light source plane $\left(x^{\prime}, y^{\prime}, z^{\prime}\right)$ to the receiving plane $(x, y, z)$.

In rectangular coordinates $\left(x^{\prime}, y^{\prime}, z^{\prime}\right)$ and at light source plane, we consider two position power Gaussian vortexes that have the same amplitude at their waist [26]

$$
E\left(x^{\prime}, y^{\prime}\right)=\left(x^{\prime} \pm i y^{\prime}\right)^{l_{0}} \exp \left(-\frac{x^{\prime 2}+y^{\prime 2}}{w_{0}^{2}}\right), x^{\prime}, y^{\prime}>0
$$

where " + " represents right- spin vortex, " - " represents the left—spin vortex, $l_{0}$ is the topological charge of vortex, and $w_{0}$ is the waist of these vortexes. Essentially, $\left(x^{\prime} \pm i y^{\prime}\right)^{l_{0}}$ is a transmission factor and is a position power vortex. The position power vortex can be produced from "fork" hologram [27].

In the cylindrical coordinate system $\left(r^{\prime}, \theta^{\prime}, z^{\prime}\right)$ these two position power Gaussian vortexes can be rewritten as follows

$$
E\left(r^{\prime}, \theta^{\prime}, 0\right)=r^{l_{0}} \exp \left(-\frac{r^{\prime 2}}{w_{0}^{2}} \pm i l_{0} \theta^{\prime}\right) \theta^{\prime}>0
$$

where $r^{\prime}=\sqrt{x^{\prime 2}+y^{\prime 2}}$, and $\theta^{\prime}=\arctan \left(y^{\prime} / x^{\prime}\right)$.

Now, according to Equation (8), we form a coaxial double-position power Gaussian vortex with a normalized field and opposite helices in the source plane $z^{\prime}=0$, as shown in the following equation (see Appendix A)

$$
E\left(r^{\prime}, \theta^{\prime}, 0\right)=N\left[\frac{1-|\alpha|}{2} r^{\prime l_{0}} \exp \left(-\frac{r^{\prime 2}}{w_{0}^{2}}-i l_{0} \theta^{\prime}\right)+\frac{1+|\alpha|}{2} r^{\prime l_{0}} \exp \left(-\frac{r^{\prime 2}}{w_{0}^{2}}+i l_{0} \theta^{\prime}\right)\right]
$$


where $N=\left\{\pi\left[(1+|\alpha|)^{2}+(1-|\alpha|)^{2}\right] 2^{-l_{0}-3} w_{0}^{2 l_{0}+2} \gamma\left(l_{0}+1, \frac{D^{2}}{2 w_{0}^{2}}\right)\right\}^{-1 / 2}$ is normalization coefficient, $D$ is the diameter of the receiver, $\gamma\left(l_{0}+1, \frac{D^{2}}{2 w_{0}^{2}}\right)$ is the incomplete gamma function, and $\alpha$ denotes a dimensionless structural parameter. When $|\alpha|=1$, the beam degenerates into left spin or right spin single-position power Gaussian vortex, respectively; when $|\alpha| \neq 1$, the beam is a coaxial double-position power Gaussian vortex with reverse helix and different amplitude, and when $|\alpha|=0$, the beam is a coaxial double-position power Gaussian vortex with inverse spiral and equal amplitude.

The field of the coaxial double-position power Gaussian vortex in free space at any receiving lane $x^{\prime}=x, y^{\prime}=y, z^{\prime}=z$ is given by (see Appendix $B$ )

$$
\begin{aligned}
E(r, \theta, z) & =-\frac{i k}{2 N z} \exp \left[i\left(k z+\frac{k r^{2}}{2 z}\right)\right]\left[(1-|\alpha|)(-i)^{l_{0}} e^{-i l_{0} \theta}+(1+|\alpha|)(i)^{l_{0}} e^{i l_{0} \theta}\right] \\
& \times \frac{z k^{l_{0}} w_{0}^{2\left(l_{0}+1\right)} r_{0}^{l_{0}}}{\left(2 z-i k w_{0}^{2}\right)^{l_{0}+1} 1} F_{1}\left(l_{0}+1 ; l_{0}+1 ;-\frac{k^{2} r^{2} w_{0}^{2}}{2 z\left(2 z-i k w_{0}^{2}\right)}\right)
\end{aligned}
$$

where $\Gamma\left(\frac{v+\mu+1}{2}\right)$ is gamma function, and ${ }_{1} F_{1}\left(\frac{v+\mu+1}{2} ; v+1 ;-\frac{b^{2}}{4 a}\right)$ is confluent hypergeometric function. $k$ is the wave number, and $\lambda$ is the wavelength of light in the vacuum.

In weak turbulence and absorbent seawater and by Rytov approximation [25], the field of the RCDPPGV is expressed as (see Appendix C)

$$
\begin{aligned}
\widetilde{E}(r, \theta, z)= & \frac{\pi\left(n_{i}-i n_{r}\right)}{N \lambda} i^{l_{0}} e^{i \frac{2 \pi}{\lambda}\left(n_{r}+i n_{i}\right)\left(z+\frac{r^{2}}{2 z}\right)+\psi(r, \theta, z)}\left[(1-|\alpha|)(-1)^{l_{0}} e^{-i l_{0} \theta}+(1+|\alpha|) e^{i l_{0} \theta}\right] \\
& \times \frac{\left[\frac{2 \pi}{\lambda}\left(n_{r}+i n_{i}\right)\right]^{l_{0}} w_{0}^{2\left(l_{0}+1\right)} r^{l_{0}}}{\left[2 z+\frac{2 \pi w_{0}^{2}}{\lambda}\left(n_{i}-i n_{r}\right)\right]^{l_{0}+1}} F_{1}\left(l_{0}+1 ; l_{0}+1 ;-\frac{\left[\frac{2 \pi}{\lambda}\left(n_{r}+i n_{i}\right)\right]^{2} r^{2} w_{0}^{2}}{4 z^{2}+\frac{4 \pi}{\lambda} z\left(n_{i}-i n_{r}\right) w_{0}^{2}}\right)
\end{aligned}
$$

where $\psi(r, \theta, z)$ represents the complex phase disturbance caused by seawater turbulence.

\section{Bit Error Rate of the OAM Link and Channel Capacity}

In this section, the series expansion method of RCDPPGV random spiral mode is used to obtain the mathematical model of bit error rate and channel capacity.

\subsection{Bit Error Rate of OAM Links}

To understand the OAM mode redistribution generated by turbulent seawater disturbance, we expanded the disturbed field into a series of the spiral harmonic exp $(i l \theta)[18,19]$

$$
\widetilde{E}(r, \theta, z)=\frac{1}{\sqrt{2 \pi}} \sum_{l=-\infty}^{\infty} a_{l}(r, z) e^{i l \theta}
$$

with

$$
a_{l}(r, z)=\frac{1}{\sqrt{2 \pi}} \int_{0}^{2 \pi} E(r, \theta, z) e^{-i l \theta} d \theta
$$

Note that $l \neq l_{0}$ is the topological charge of an OAM crosstalk mode.

The ensemble average of $\left|a_{l}\right|^{2}$ represents the condition probability distribution of OAM modes in turbulent as well as absorbent seawater and at receiving plane, which has the form of

$$
\begin{aligned}
p_{l / l_{0}}(r) & =\left\langle\left|a_{l}(r, z)\right|^{2}\right\rangle \\
& =\frac{1}{2 \pi} \mid \int_{0}^{2 \pi} \int_{0}^{2 \pi} E(r, \theta, z) E^{*}(r, \theta, z) \exp \left[-i l\left(\theta-\theta^{\prime}\right)\right] \\
& \times \exp \left[-2 r^{2}\left[1-\cos \left(\theta-\theta^{\prime}\right)\right] / \rho_{o c}^{2}\right] d \theta d \theta^{\prime} \mid
\end{aligned}
$$


where * denotes the complex conjugate, and $\rho_{o c}$ is the spatial coherence radius of waves propagating in isotropic and turbulent as well as absorbent seawater and is given by [13]

$$
\begin{aligned}
& \rho_{o c}^{-2}=\frac{2.511 \times 10^{-7} \varepsilon^{-1 / 3} \chi_{T}\left(n_{r}^{2}+n_{i}^{2}\right) z}{\lambda^{2}}\left[\kappa_{0}^{1 / 3} U\left(2 ; \frac{7}{6} ; \frac{\kappa_{0}^{2} \eta^{2}}{R_{T}^{2}}\right)\right. \\
& +4.6 \eta^{2 / 3} \kappa_{0} \Gamma\left(\frac{7}{3}\right) U\left(\frac{7}{3} ; \frac{3}{2} ; \frac{\kappa_{0}^{2} \eta^{2}}{R_{T}^{2}}\right)+\frac{1}{\omega^{2}} \kappa_{0}^{1 / 3} U\left(2 ; \frac{7}{6} ; \frac{\kappa_{0}^{2} \eta^{2}}{R_{S}^{2}}\right) \\
& +\frac{4.6}{\omega^{2}} \eta^{2 / 3} \kappa_{0} \Gamma\left(\frac{7}{3}\right) U\left(\frac{7}{3} ; \frac{3}{2} ; \frac{\kappa_{0}^{2} \eta^{2}}{R_{S}^{2}}\right)-\frac{2}{\omega} \kappa_{0}^{1 / 3} U\left(2 ; \frac{7}{6} ; \frac{\kappa_{0}^{2} \eta^{2}}{R_{T S}^{2}}\right) \\
& \left.-\frac{9.2}{\omega} \eta^{2 / 3} \kappa_{0} \Gamma\left(\frac{7}{3}\right) U\left(\frac{7}{3} ; \frac{3}{2} ; \frac{\kappa_{0}^{2} \eta^{2}}{R_{T S}^{2}}\right)\right] .
\end{aligned}
$$

Substituting Equation (9) into Equation (12), through integration operations, we obtain the probability distribution of OAM modes (see Appendix D)

$$
\begin{aligned}
& p_{l / l_{0}}(r)=\frac{2 \pi^{3}\left(n_{r}^{2}+n_{i}^{2}\right)\left[\left(\frac{2 \pi}{\lambda}\right)^{2}\left(n_{r}^{2}+n_{i}^{2}\right)\right]^{l_{0}} w_{0}^{4\left(l_{0}+1\right)} r^{2 l_{0}}}{N_{2} \lambda^{2}\left[\left(2 z+\frac{2 \pi}{\lambda} n_{i} w_{0}^{2}\right)^{2}+\left(n_{r} \frac{2 \pi}{\lambda} w_{0}^{2}\right)^{2}\right]^{l_{0}+1}} \\
& \quad \times\left|{ }_{1} F_{1}\left(l_{0}+1 ; l_{0}+1 ;-\frac{\left[\frac{2 \pi}{\lambda}\left(n_{r}+i n_{i}\right)\right]^{2} r^{2} w_{0}^{2}}{4 z^{2}+\frac{4 \pi}{\lambda} z\left(n_{i}-i n_{r}\right) w_{0}^{2}}\right)\right|^{2} \exp \left[-\frac{2 \pi}{\lambda} n_{i}\left(z+\frac{r^{2}}{2 z}\right)-\frac{2 r^{2}}{\rho_{o c}^{2}}\right] \\
& \times\left|\left[(|\alpha|-1)^{2} I_{l+l_{0}}\left(\frac{2 r^{2}}{\rho_{o c}^{2}}\right)+(|\alpha|+1)^{2} I_{l-l_{0}}\left(\frac{2 r^{2}}{\rho_{o c}^{2}}\right)\right]\right|
\end{aligned}
$$

The receiving probability of OAM mode carried by RCDPPGV and measured by the detector with detection diameter $D$ can be expressed as

$$
P_{l / l_{0}}=2 \pi \int_{0}^{D / 2} p_{l / l_{0}}(r) r d r
$$

In addition, for sufficiently large transmit power, the signal-to-noise ratio (SNR) of OAM channel is approximately [28]

$$
\operatorname{SNR}\left(l, l_{0}\right)=\frac{P_{l / l_{0}, l=l_{0}}}{\sum_{l=-\infty}^{\infty} P_{l / l_{0}, l \neq l_{0}}}
$$

For signal modulation of on-off keying (OOK), based on (18), the bit error rate (BER) of OAM channels is derived as [29]

$$
\operatorname{BER}\left(l, l_{0}\right)=\frac{1}{2} \operatorname{erfc} \sqrt{\frac{\operatorname{SNR}\left(l, l_{0}\right)}{2}}
$$

where $\operatorname{erfc}(\cdot)$ denotes the complementary error function.

\subsection{Channel Capacity}

Consider a line-of-sight communication link using OAM eigenstates in the range of topological charge number $l_{0}=0,1, \ldots, M-1$ that means $M$ dimension Hilbert space for different signal values. Further, by classical Shannon information theory of a stationary discrete memory-less system [3] and Equation (17), the channel capacity of a communication link with coaxial double-position power Gaussian vortex in weakly absorbent seawater can be given by [3]

$$
C=\log _{2} M+\frac{1}{M} \sum_{l=-\infty}^{\infty} \sum_{l_{0}=0}^{M-1} P_{l \mid l_{0}}\left[\log _{2} P_{l \mid l_{0}}-\log _{2} \sum_{l_{0}} P_{l \mid l_{0}}\right] .
$$


with the entropy of the signal vortex mode $l_{0}$

$$
\max _{\left\{P_{l_{0}}\right\}} H_{l_{0}}=\log _{2} M,
$$

and conditional entropy of the $l$ mode for given signal mode $l_{0}$

$$
\max _{\left\{P_{l_{0}}\right\}} H_{l_{0} \mid l}=-\frac{1}{M} \sum_{l=-\infty}^{\infty} \sum_{l_{0}=0}^{M-1} P_{l \mid l_{0}}\left[\log _{2} P_{l \mid l_{0}}-\log _{2} \sum_{l_{0}} P_{l \mid l_{0}}\right]
$$

\section{Numeric Analysis}

To explore the influence of beam structure parameters and seawater parameters on BER and the channel capacity of OAM links in isotropic turbulent as well as absorbent seawater, in this section, we numerically study the BER and channel capacity of the link with a RCDPPGV carrier. In the following analysis, we set the calculation parameters as: $|\alpha|=1, w_{0}=0.05 \mathrm{~m}, D=0.1 \mathrm{~m}, z=100 \mathrm{~m}, \lambda=561 \mathrm{~nm}, \chi_{t}=10^{-8} \mathrm{~K}^{2} / \mathrm{s}, \varepsilon=10^{-4} \mathrm{~m}^{2} / \mathrm{s}^{3}$, $\eta=1 \mathrm{~mm}, l_{0}=1, L_{0}=10 \mathrm{~m}, \omega=-4, M=5, n_{r}=1.34$, and $n_{i}=0.6741 \times 10^{-10}$, unless other variable parameters are specified in calculation. Moreover, in the following numerical analysis, we first analyze the influence of system parameters and turbulent seawater on the BER of OAM channels and, then, analyze the influence of system parameters and turbulent seawater on channel capacity.

\subsection{Effects of Seawater Turbulence and Beam Parameters on the BER}

Figure 2 shows variation curve of the BER of the random double-Gaussian optical vortex carrier communication link with the dissipation rate of the mean-squared temperature $\chi_{T}$ and topological charge of the RCDPPGV vortex. The variation curve shows that with the increase in transmission distance $z$, the BER of this link increases. When the transmission distance is less than $50 \mathrm{~m}, \mathrm{BER}$ increases rapidly and, then, tends to be flat. Additionally, a larger $l_{0}$ means a higher BER. The physical reason for this result is that when the topological charge (orbital angular momentum quantum number) of OAM signal mode is larger, the OAM energy level of the signal is also higher. Therefore, the probability of transition form to another OAM energy level is also higher when it is disturbed.

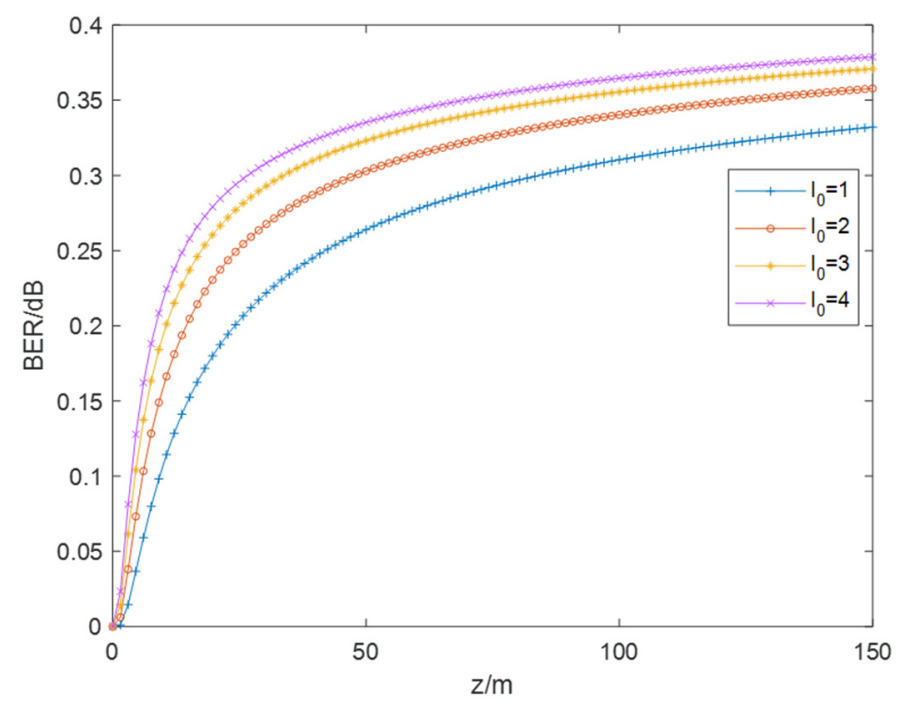

Figure 2. BER of the coaxial double-position power Gaussian vortex carrier communication link versus dissipation rate of the mean-squared temperature $\chi_{T}$ for different topological charge of vortex.

Under certain topological charge of vortex laser, we use Figure 3 to show the variation curve of BER of random double-Gaussian optical vortex carrier communication link with dimensionless parameter $\alpha$. From Figure 2, we can see that BER has two minimum values 
at $|\alpha|=1$. This result indicates that the link with a single-position power Gaussian vortex has minimum BER.

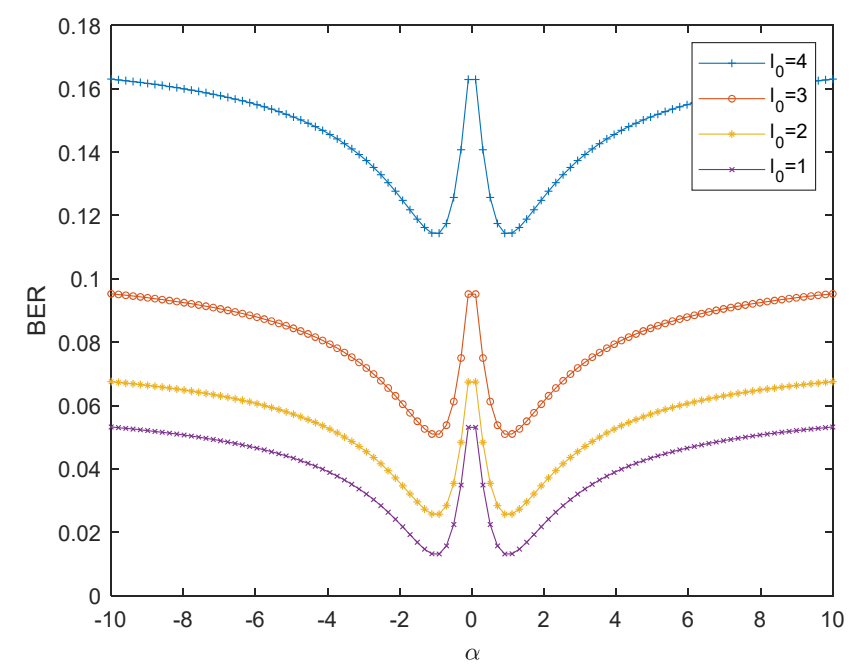

Figure 3. BER of the coaxial double-position power Gaussian vortex carrier communication link versus dimensionless parameter $|\alpha|$ for different topological charge of vortex.

In Figure 4, we investigate the effects of the $\varepsilon$ and $l_{0}$ on the BER of RCDPPGV carrier link for given topological charge of vortex. From Figure 3, we can see that the value of the BER decreases with an increase in the $\varepsilon$. The reason for this phenomenon is the increase in $\varepsilon$, the turbulence strength of seawater decreases, and the influence of turbulence on the transmission vortex decreases.

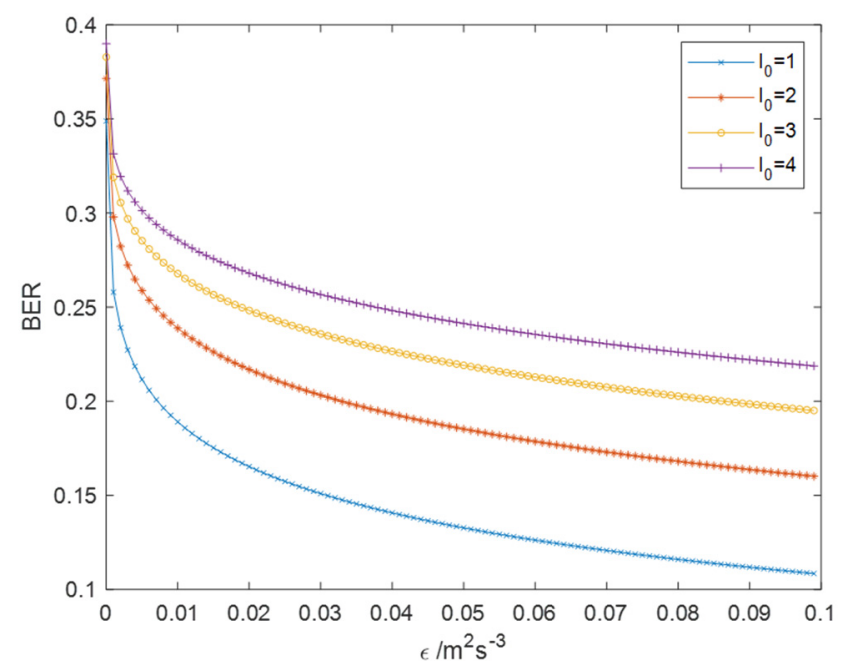

Figure 4. BER of the coaxial double-position power Gaussian vortex carrier communication link versus dissipation rate of kinetic energy per unit mass of fluid $\varepsilon$ for different topological charge of vortex.

In Figure 5, we investigate the effects of dissipation rate of the mean-squared temperature $\chi_{T}$ on the channel capacity of the RCDPPGV carrier link for the given topological charge of vortex. It is clear from Figure 5 that the BER of the channel capacity increases with increasing $\chi_{T}$. The reason for this result comes from that with the increase in $\chi_{T}$, the turbulence strength of seawater increases, and the influence of turbulence on the transmission vortex increases. 


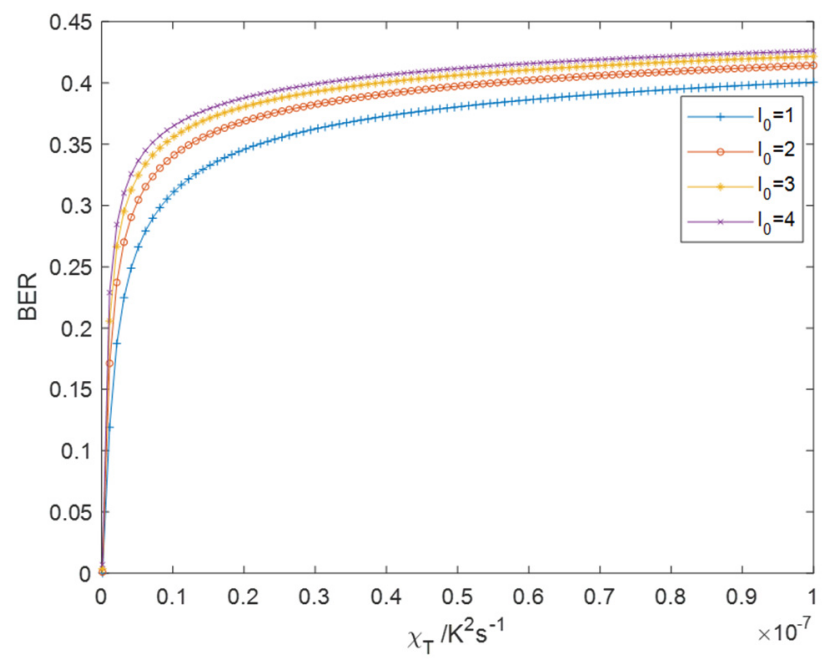

Figure 5. BER of the coaxial double-position power Gaussian vortex carrier communication link versus dissipation rate of the mean-squared temperature $\chi_{T}$ for different topological charge of vortex.

\subsection{Effects of Seawater Turbulence and Beam Parameters on Channel Capacity}

Figure 6 illustrates the impact of the dimensionless structural parameters $\alpha$ on the channel capacity of the RCDPPGV carrier with different values of the channel numbers M. Figure 6 shows that the channel capacity in the $[-10,10]$ interval of $\alpha$ is a nonlinear function of $\alpha$ and reaches two maximum values at $|\alpha|=1$. The difference value between the maximum channel capacity of each link increases with the number of OAM channels M. However, with the decrease in $|\alpha|$ value, channel capacity tends to be constant, this constant is channel capacity at $|\alpha|=0$. This result indicates that the link with singleposition power Gaussian vortex has maximum channel capacity. Similar to any other type of beam [9-22], Figure 6 shows that the channel capacity also increases with the increase in channel number.

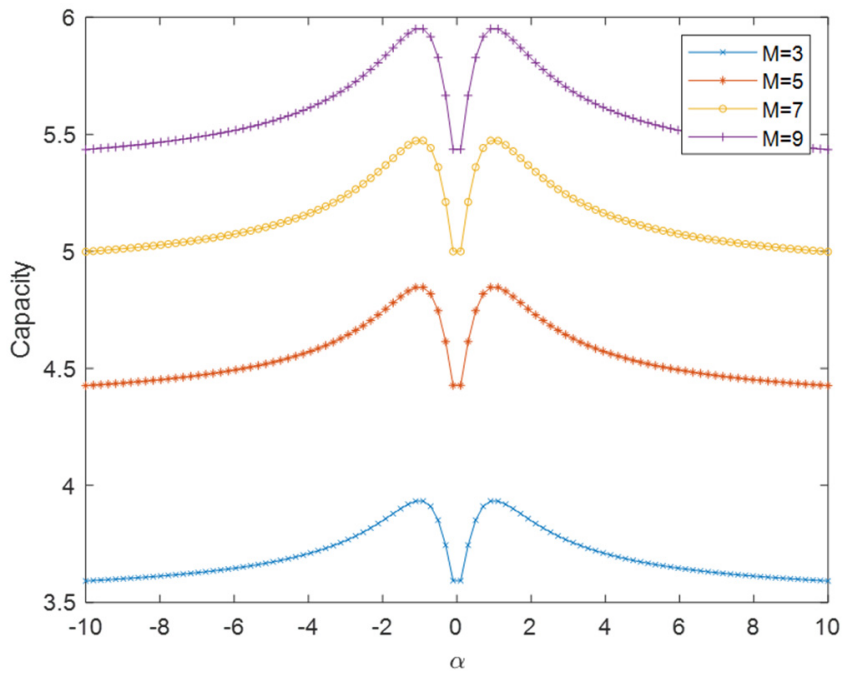

Figure 6. Channel capacity of the link with the coaxial double-position power Gaussian vortex carrier versus dimensionless parameter $|\alpha|=1$ for different channel numbers $M$.

Figure 7 shows that channel capacity decays rapidly as absorption increases. Seawater absorption has a great influence on the channel capacity, so in the detector design of the underwater communication system, it should take into account the inconsistency between the absorption of the offshore area and the open sea area. 


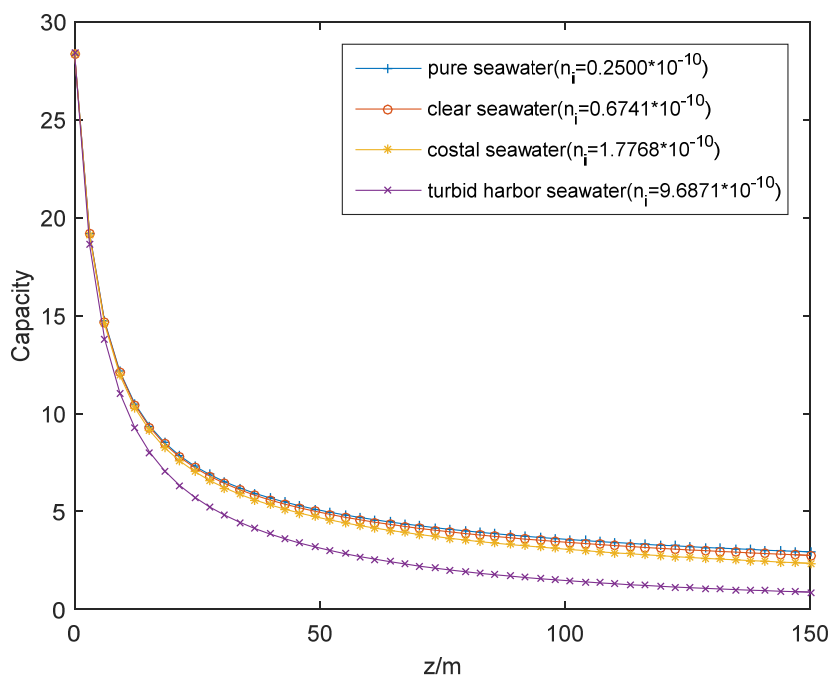

Figure 7. Channel capacity of the link with the random double-position power Gaussian vortex carrier and wavelength $\lambda=561 \mathrm{~nm}$ versus pure seawater, clear seawater, costal seawater, and turbid harbor seawater.

Figure 8 shows the channel capacity of the RCDPPGV carrier versus the initial beam width $w_{0}$ for different values of received diameter $\mathrm{D}$. We can find that the channel capacity of the link with RCDGV carrier decreases with increase in the $w_{0}$. This phenomenon can be explained as follows: when the initial beam size $w_{0}$ is larger, the difference of turbulent seawater within the beam diameter is also larger. Of course, the wave front distortion of the transmitting beam is also large, so that the difference between signal probability and crosstalk probability tends to zero [30]. Figure 8 also shows that the channel capacity of the link with RCDPPGV carrier increases with an increase in D. This result can be explained by the following reasons: because the channel of OAM mode with higher topological charge is farther away from the axis of communication link [1,26], the receiver with the larger receiving aperture can receive the information of the OAM mode channel with higher topological charge.

In Figure 8, we investigate the effects of the temperature-salinity contribution ratios and different wavelength $\lambda$ on the channel capacity of the RCDPPGV carrier in the "seawater light transmission window". Similar to any other type of beam [9-22], Figure 8 shows that the channel capacity of the RCDPPGV carrier increases with increasing $\lambda$. This is because the long-wavelength beam has lower scintillations [25]. As $\omega$ increases, channel capacity decreases. This study is similar to studies for the single vortex information carrier [9-22], and salinity fluctuations have a greater impact on the channel capacity with a larger number of channels.

In Figure 9, we investigate the effects of the outer scale of turbulence $L_{0}$ and the inner scale of turbulence $\eta$ on the channel capacity of RCDPPGV carrier. From Figure 5, we can see that the value of the channel capacity increases with an increase in $\eta$ and a decrease in $L_{0}$. This shows that the channel capacity increases with the increase in the inner scale of turbulence. According to the turbulence theory, with the increase in the inner scale of turbulence, the uniform area in the seawater increases when the beam passes through the channel, that is, the influence of the channel on the transmitted beam decreases. We can also find that the channel capacity decreases with the increase in the outer scale, but the range of change is small. The smaller the outer scale of turbulence is, the less wave front distortion will be generated, so the crosstalk probability will be smaller. The reasons for this phenomenon are as follows: When the turbulent outer scale increases, the deflection of light ray in the beam is growing, and the frequency of light rays going through different paths also increases. As a result, the optical path difference between the light rays increases, and the wave front distortion of the beam increases accordingly. However, because the 
refractive index difference is small in the outer scale of turbulence, the wave front distortion of the beam increases but not much.

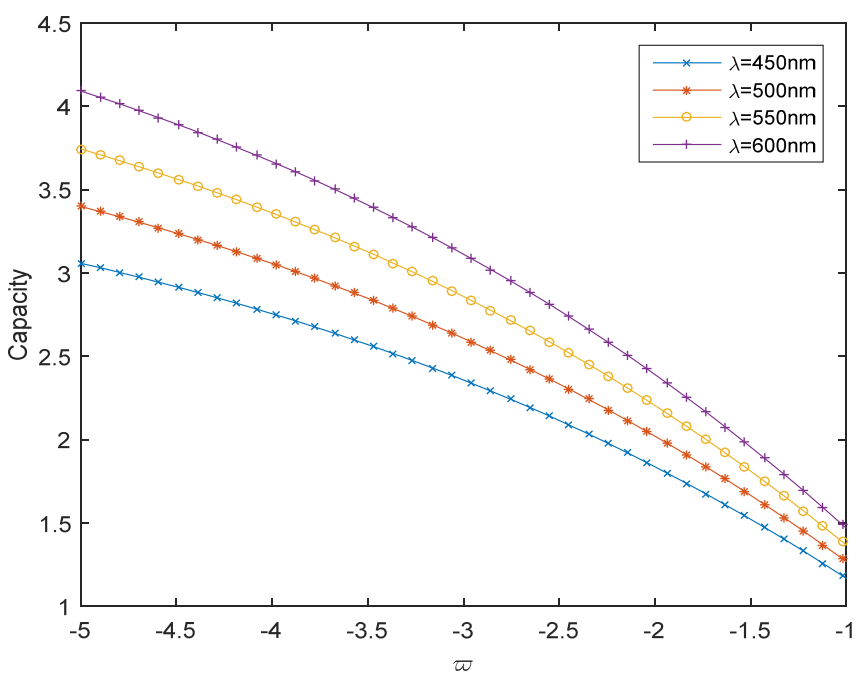

Figure 8. Channel capacity of the link with the random double-position power Gaussian vortex carrier communication system versus temperature-salinity contribution ratios and different values of wavelength $\lambda$.

In Figure 10, we investigate the effects of dissipation rate of kinetic energy per unit mass of fluid and dissipation rate of the mean-squared temperature $\chi_{t}$ on the channel capacity of the RCDPPGV carrier. It is clear from Figure 6 that the magnitude of the channel capacity decreases with increasing $\chi_{t}$ and decreasing $\varepsilon$. The reason for this result comes from that with the increase in $\chi_{t}$ and the decrease in $\varepsilon$, the turbulence of seawater increases, and the influence of turbulence on the beam increases [31].

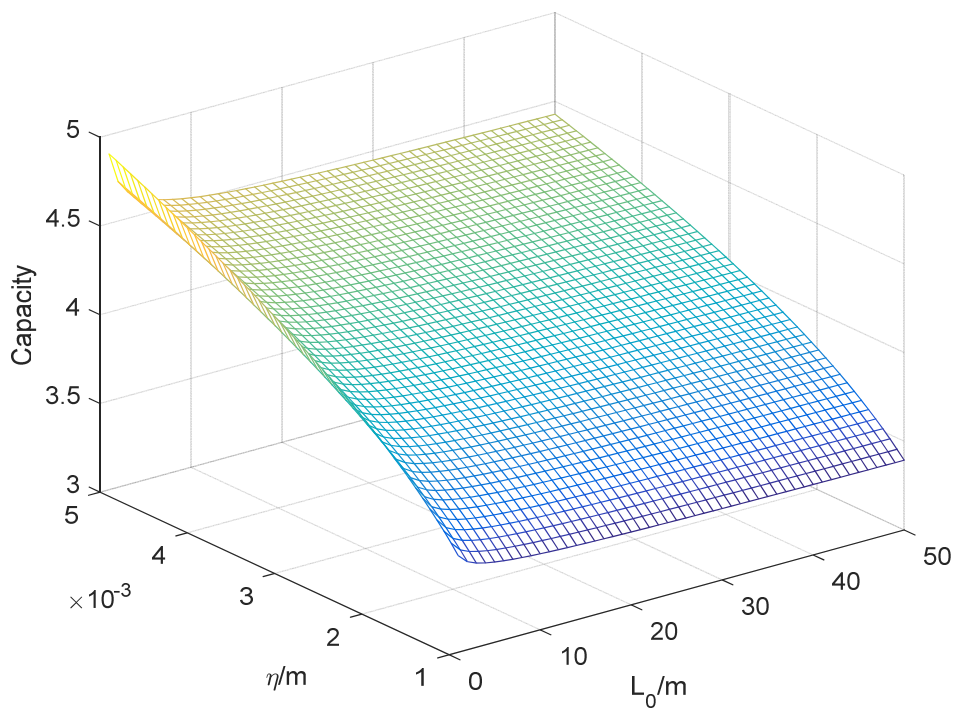

Figure 9. Channel capacity of the link with the random double-position power Gaussian vortex carrier optics communication system versus the inner scale of turbulence $\eta$ and the outer scale of turbulence $L_{0}$. 


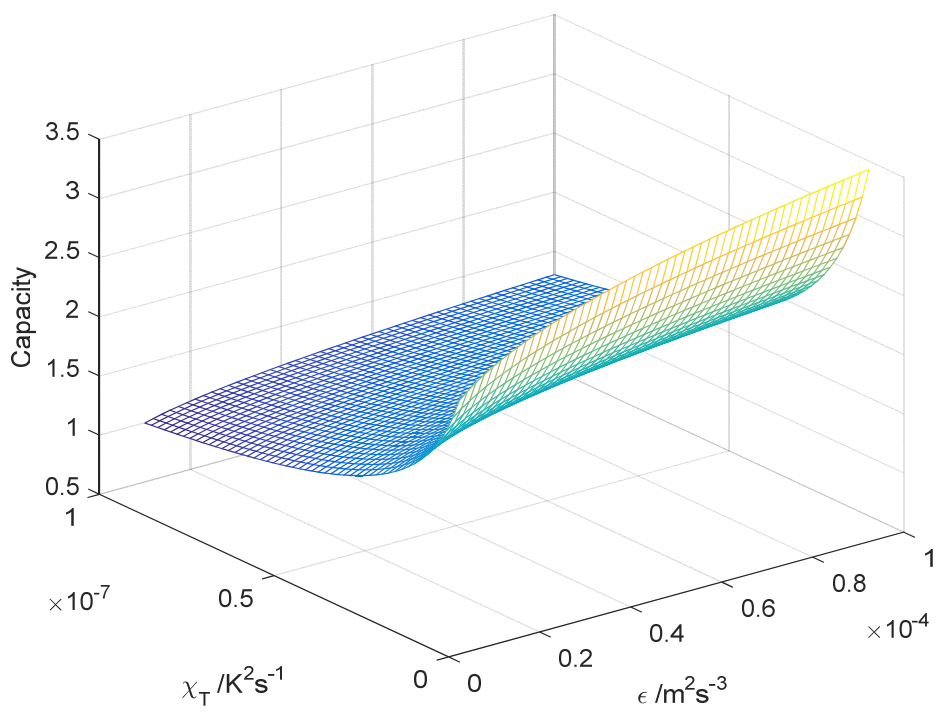

Figure 10. Channel capacity of the link with the random double-position power Gaussian vortex carrier optics communication system versus dissipation rate of kinetic energy per unit mass of fluid $\varepsilon$ and dissipation rate of the mean-squared temperature $\chi_{T}$.

\section{Conclusions}

We focused on the propagation properties of OAM modes carried by the RCDPPGV carrier in absorptive, isotropic, and weak turbulent ocean link and derived the channel capacity of this link. The results show that seawater absorption has a great influence on the channel capacity of the optics communication link, and the larger the seawater absorption, the faster the absorption of channel capacity with the transmission distance. With the optics communication link with single-position power Gaussian vortex carrier, the channel capacity of the link reached an extreme value. The channel capacity increases with the increase in the $\varepsilon$ and $\eta$, and the change in $L_{0}$ has little effect on the channel capacity. The channel capacity decreases with increasing $\omega$ and $\chi_{t}$. The large number of OAM channels $M$ leads to higher channel capacity. The channel capacity decreases with the increase in initial beam width and the decrease in D. Our results also show that with the increase in transmission distance $z$, the topological charge of vortex, and $\varepsilon$, the BER of this link increases; however, the BET of the link increases with the increase in $\chi_{T}$ and is minimum at $\alpha=1$ or -1 . Our paper also points out that we need to further study the influence of the helical direction of non-coaxial multi-helical combination vortices on the channel capacity of the communication link.

Author Contributions: Conceptualization, Y.Z. (Yixin Zhang) and Q.Y.; methodology, Y.Z. (Yixin Zhang); software, Q.Y.; validation, Y.Z. (Yixin Zhang), Q.Y. and Y.Z. (Yun Zhu); formal analysis, Q.Y.; investigation, Q.Y.; resources, Y.Z. (Yixin Zhang); data curation, Q.Y.; writing—original draft preparation, Y.Z. (Yun Zhu); writing - review and editing, Y.Z. (Yixin Zhang); visualization, Q.Y.; supervision, Y.Z. (Yixin Zhang); project administration, Y.Z. (Yixin Zhang); funding acquisition, Y.Z. (Yixin Zhang). All authors have read and agreed to the published version of the manuscript.

Funding: This work was supported by the National Natural Science Foundation of China (61871202).

Institutional Review Board Statement: Not applicable.

Informed Consent Statement: Not applicable.

Data Availability Statement: Not applicable.

Conflicts of Interest: The authors declare no conflict of interest. 


\section{Appendix A}

According to Equation (8), we form coaxial double-position power Gaussian vortex with the normalization field and opposite spirals at the source plane $z=0$ as follows

$$
E\left(r^{\prime}, \theta^{\prime}, 0\right)=\left[\frac{1-|\alpha|}{2} r^{\prime l_{0}} \exp \left(-\frac{r^{\prime 2}}{w_{0}^{2}}-i l_{0} \theta^{\prime}\right)+\frac{1+|\alpha|}{2} r^{\prime l_{0}} \exp \left(-\frac{r^{\prime 2}}{w_{0}^{2}}+i l_{0} \theta^{\prime}\right)\right]
$$

Consider the following normalization relations

$$
\int_{0}^{D / 2} \int_{0}^{2 \pi} E\left(r^{\prime}, \theta^{\prime}, 0\right) E^{*}\left(r^{\prime}, \theta^{\prime}, 0\right) d \theta r d r=1
$$

Applying the integral relation [32] $\int_{0}^{u} x^{m} e^{-b x^{2}} d x=\frac{\gamma\left(\frac{m+1}{2}, b u^{2}\right)}{2 b^{\frac{m+1}{2}}}$ in the above equation, the normalization field and opposite spirals at the source plane $z=0$

$$
E\left(r^{\prime}, \theta^{\prime}, 0\right)=N\left[\frac{1-|\alpha|}{2} r^{\prime l_{0}} \exp \left(-\frac{r^{\prime 2}}{w_{0}^{2}}-i l_{0} \theta^{\prime}\right)+\frac{1+|\alpha|}{2} r^{\prime l_{0}} \exp \left(-\frac{r^{\prime 2}}{w_{0}^{2}}+i l_{0} \theta^{\prime}\right)\right]
$$

with normalization coefficient

$$
N=\left\{2^{-l_{0}-3} \pi w_{0}^{2 l_{0}+2}\left[(1+|\alpha|)^{2}+(1-|\alpha|)^{2}\right] \gamma\left(l_{0}+1, \frac{D^{2}}{2 w_{0}^{2}}\right)\right\}^{-1 / 2}
$$

\section{Appendix B}

By Huygens-Fresnel diffraction integral Equation (9), the field of the coaxial doubleposition power Gaussian vortex in free space at the receiving plane is given by $[31,33]$

$$
\begin{aligned}
E(r, \theta, z) & =-\frac{i k}{2 \pi z} \exp \left[i\left(k z+\frac{k r^{2}}{2 z}\right)\right] \int_{0}^{\infty} \int_{0}^{2 \pi} E\left(r^{\prime}, \theta^{\prime}, 0\right) \\
& \times \exp \left\{i \frac{k}{2 z}\left[r^{\prime 2}-2 r r^{\prime} \cos \left(\theta-\theta^{\prime}\right)\right]\right\} r^{\prime} d r^{\prime} d \theta^{\prime} \\
& =-\frac{i k}{2 \pi N z} e^{i\left(k z+\frac{k r^{2}}{2 z}\right)} \int_{0}^{\infty} r^{\prime l_{0}+1} e^{-\left(\frac{1}{w_{0}^{2}}-\frac{i k}{2 z}\right) r^{\prime 2}} \\
& \left\{\frac{|\alpha|-1}{2} \int_{0}^{2 \pi} e^{-i\left[l_{0} \theta^{\prime}+\frac{k r r^{\prime}}{z} \cos \left(\theta-\theta^{\prime}\right)\right]} d \theta^{\prime}+\frac{|\alpha|+1}{2} \int_{0}^{2 \pi} e^{-i\left[-l_{0} \theta^{\prime}+\frac{k r r^{\prime}}{z} \cos \left(\theta-\theta^{\prime}\right)\right]} d \theta^{\prime}\right\} d r^{\prime}
\end{aligned}
$$

Consider integrals [32]

$$
\begin{aligned}
& \int_{0}^{2 \pi} e^{-i\left[l_{0} \theta^{\prime}+\frac{k r r^{\prime}}{z} \cos \left(\theta-\theta^{\prime}\right)\right]} d \theta^{\prime}=(-i)^{l_{0}} 2 \pi J_{l_{0}}\left(\frac{k r r^{\prime}}{z}\right) e^{-i l_{0} \theta} \\
& \int_{0}^{2 \pi} e^{-i\left[-l_{0} \theta^{\prime}+\frac{k r r^{\prime}}{z} \cos \left(\theta-\theta^{\prime}\right)\right]} d \theta^{\prime}=(i)^{l_{0}} 2 \pi J_{l_{0}}\left(\frac{k r r^{\prime}}{z}\right) e^{-i l_{0} \theta}
\end{aligned}
$$

Equation (A1) becomes

$$
\begin{aligned}
E(r, \theta, z) & =-\frac{i k}{2 N z} \exp \left[i\left(k z+\frac{k r^{2}}{2 z}\right)\right] \\
& \times\left\{\left[(1-|\alpha|)(-i)^{l_{0}} e^{-i l_{0} \theta}+(1+|\alpha|)(i)^{l_{0}} e^{i l_{0} \theta}\right]\right. \\
& \left.+\int_{0}^{\infty} r^{\prime l_{0}+1} \exp \left[-\left(\frac{1}{w_{0}^{2}}-\frac{i k}{2 z}\right) r^{\prime 2}\right] J_{l_{0}}\left(\frac{k r r^{\prime}}{z}\right) d r^{\prime}\right\}
\end{aligned}
$$

Further, consider the following integrals further [32]

$$
\int_{0}^{\infty} r^{\prime \mu} e^{-a r^{\prime 2}} J_{v}\left(b r^{\prime}\right) d r^{\prime}=\frac{b^{v} \Gamma\left(\frac{v+\mu+1}{2}\right)}{2^{v+1} a^{\frac{1}{2}(v+\mu+1)} \Gamma(v+1)}{ }_{1} F_{1}\left(\frac{v+\mu+1}{2} ; v+1 ;-\frac{b^{2}}{4 a}\right), \operatorname{Re} a>0
$$


Equation (A4) becomes

$$
\begin{aligned}
E(r, \theta, z) & =-\frac{i k}{2 N z} \exp \left[i\left(k z+\frac{k r^{2}}{2 z}\right)\right]\left[(1-|\alpha|)(-i)^{l_{0}} e^{-i l_{0} \theta}+(1+|\alpha|)(i)^{l_{0}} e^{i l_{0} \theta}\right] \\
& \times \frac{z k^{l_{0}} w_{0}^{2\left(l_{0}+1\right)} r_{0}^{l_{0}}}{\left(2 z-i k w_{0}^{2}\right)^{l_{0}+1}} F_{1}\left(l_{0}+1 ; l_{0}+1 ;-\frac{k^{2} r^{2} w_{0}^{2}}{2 z\left(2 z-i k w_{0}^{2}\right)}\right)
\end{aligned}
$$

where $\Gamma\left(\frac{v+\mu+1}{2}\right)$ is gamma function, and ${ }_{1} F_{1}\left(\frac{v+\mu+1}{2} ; v+1 ;-\frac{b^{2}}{4 a}\right)$ is confluent hypergeometric function. $k$ is the wave number, and $\lambda$ is the wavelength of light in the vacuum.

\section{Appendix C}

In weak turbulence and extinction seawater, by using the Rytov approximation [25] and Equation (10), the field of random coaxial double-position power Gaussian vortex (RCDPPGV) laser is expressed as

$$
\begin{aligned}
E(r, \theta, z) & =-\frac{i k}{2 N z} e^{i\left(k z+\frac{k r^{2}}{2 z}\right)+\psi(r, \theta, z)}\left[(1-|\alpha|)(-i)^{l_{0}} e^{-i l_{0} \theta}+(1+|\alpha|)(i)^{l_{0}} e^{i l_{0} \theta}\right] \\
& \times \frac{z k_{0}^{l_{0}} w_{0}^{2\left(l_{0}+1\right)} r^{l_{0}}}{\left(2 z-i k w_{0}^{2}\right)^{l_{0}+1}} F_{1}\left(l_{0}+1 ; l_{0}+1 ;-\frac{k^{2} r^{2} w_{0}^{2}}{2 z\left(2 z-i k w_{0}^{2}\right)}\right)
\end{aligned}
$$

where $k=\frac{2 \pi}{\lambda}\left(n_{r}+i n_{i}\right)$, and $\psi(r, \theta, z)$ represents the complex phase disturbance caused by seawater turbulence.

In Equation (A11), the wave number of seawater $k$ is substituted, and Equation (A11) is rewritten as

$$
\begin{aligned}
\widetilde{E}(r, \theta, z) & =\frac{\pi\left(n_{i}-i n_{r}\right)}{N \lambda z} i_{0} e^{\frac{2 \pi}{\lambda}\left(i n_{r}-n_{i}\right)\left(z+\frac{r^{2}}{2 z}\right)+\psi(r, \theta, z)}\left[(1-|\alpha|)(-1)^{l_{0}} e^{-i l_{0} \theta}+(1+|\alpha|) e^{i l_{0} \theta}\right] \\
& \times \frac{z\left[\frac{2 \pi}{\lambda}\left(n_{r}+i n_{i}\right)\right]^{l_{0}} w_{0}^{2\left(l_{0}+1\right)} r_{0}{ }^{l_{0}}}{\left[2 z+\frac{2 \pi}{\lambda}\left(n_{i}-i n_{r}\right) w_{0}^{2}\right]^{l_{0}+1}} F_{1}\left(l_{0}+1 ; l_{0}+1 ;-\frac{\left[\frac{2 \pi}{\lambda}\left(n_{r}+i n_{i}\right)\right]^{2} r^{2} w_{0}^{2}}{4 z^{2}+\frac{4 \pi}{\lambda} z\left(n_{i}-i n_{r}\right) w_{0}^{2}}\right)
\end{aligned}
$$

\section{Appendix D}

Rewrite the condition probability distribution of OAM modes Equation (14) in turbulent as well as absorbent seawater and at the receiving plane

$$
\begin{aligned}
p\left(l / l_{0}\right) & =\left\langle\left|a_{l}(r, z)\right|^{2}\right\rangle \\
& =\frac{1}{2 \pi}\left|\int_{0}^{2 \pi} \int_{0}^{2 \pi} \widetilde{E}(r, \theta, z) \widetilde{E}^{*}(r, \theta, z) \exp \left[-i l\left(\theta-\theta^{\prime}\right)\right] d \theta d \theta^{\prime}\right|
\end{aligned}
$$

Substitute Equation (11) into Equation (A13) and make a simple arrangement to obtain the following equation

$$
\begin{aligned}
p\left(l / l_{0}\right) & =\frac{\pi\left(n_{r}^{2}+n_{i}^{2}\right)\left[\left(\frac{2 \pi}{\lambda}\right)^{2}\left(n_{r}^{2}+n_{i}^{2}\right)\right]^{l_{0}} w_{0}^{4\left(l_{0}+1\right)} r^{2 l_{0}}}{2 N^{2} \lambda^{2}\left[\left(2 z+\frac{2 \pi}{\lambda} n_{i} w_{0}^{2}\right)^{2}+\left(n_{r} \frac{2 \pi}{\lambda} w_{0}^{2}\right)^{2}\right]^{l_{0}+1}} e^{-\frac{2 \pi}{\lambda} n_{i}\left(z+\frac{r^{2}}{2 z}\right)-\frac{2 r^{2}}{\rho_{o c}^{2}}} \\
& \times\left|{ }_{1} F_{1}\left(l_{0}+1 ; l_{0}+1 ;-\frac{\left[\frac{2 \pi}{\lambda}\left(n_{r}+i n_{i}\right)\right]^{2} r^{2} w_{0}^{2}}{4 z^{2}+\frac{4 \pi}{\lambda} z\left(n_{i}-i n_{r}\right) w_{0}^{2}}\right)\right|^{2} \\
& \times \mid \int_{0}^{2 \pi} \int_{0}^{2 \pi} e^{2 r^{2} \frac{\cos \left(\theta-\theta^{\prime}\right)}{\rho_{o c}^{2}}}\left[\left(1-|\alpha|^{2}\right) e^{-i\left(l+l_{0}\right)\left(\theta-\theta^{\prime}\right)}+(-1)^{l_{0}}\left(1-|\alpha|^{2}\right) e^{-i 2 l_{0} \theta \prime} e^{i\left(l-l_{0}\right)\left(\theta-\theta^{\prime}\right)}\right. \\
& \left.+\left(1-|\alpha|^{2}\right)(-1)^{l_{0}} e^{2 i l_{0} \theta^{\prime}} e^{-i\left(l+l_{0}\right)\left(\theta-\theta^{\prime}\right)}+\left(1+|\alpha|^{2}\right) e^{-i\left(l-l_{0}\right)\left(\theta-\theta^{\prime}\right)}\right] d \theta d \theta^{\prime} \mid
\end{aligned}
$$


By integrals $\int_{0}^{2 \pi} e^{-i m \theta+x \cos (\theta-\phi)} d \theta=2 \pi e^{-i m \phi} I_{m}(x)$ and $\int_{0}^{2 \pi} e^{-i(m-n) \theta} d \theta=2 \pi \delta_{m n}$ [32], where $\delta_{m n}$ is the Kronick notation, we have the conditional probability distribution $p\left(l / l_{0}\right)$ of the OAM modes of the RCDPPGV laser

$$
\begin{aligned}
p\left(l / l_{0}\right) & =\frac{2 \pi^{3}\left(n_{r}^{2}+n_{i}^{2}\right)\left[\left(\frac{2 \pi}{\lambda}\right)^{2}\left(n_{r}^{2}+n_{i}^{2}\right)\right]^{l_{0}} w_{0}^{4\left(l_{0}+1\right)} r^{2 l_{0}}}{N^{2} \lambda^{2}\left[\left(2 z+\frac{2 \pi}{\lambda} n_{i} w_{0}^{2}\right)^{2}+\left(n_{r} \frac{2 \pi}{\lambda} w_{0}^{2}\right)^{2}\right]^{l_{0}+1}} e^{-\frac{2 \pi}{\lambda} n_{i}\left(z+\frac{r^{2}}{2 z}\right)-\frac{2 r^{2}}{\rho_{o c}^{2}}} \\
& \times\left|{ }_{1} F_{1}\left(l_{0}+1 ; l_{0}+1 ;-\frac{\left[\frac{2 \pi}{\lambda}\left(n_{r}+i n_{i}\right)\right]^{2} r^{2} w_{0}^{2}}{4 z^{2}+\frac{4 \pi}{\lambda} z\left(n_{i}-i n_{r}\right) w_{0}^{2}}\right)\right|^{2} \\
& \times\left|\left[(1-|\alpha|)^{2} I_{l+l_{0}}\left(\frac{2 r^{2}}{\rho_{o c}^{2}}\right)+(1+|\alpha|)^{2} I_{l-l_{0}}\left(\frac{2 r^{2}}{\rho_{o c}^{2}}\right)\right]\right| .
\end{aligned}
$$

where $\Gamma\left(\frac{v+\mu+1}{2}\right)$ is the gamma function, and ${ }_{1} F_{1}\left(\frac{v+\mu+1}{2} ; v+1 ;-\frac{b^{2}}{4 a}\right)$ is the confluent hypergeometric function.

\section{References}

1. Allen, L.; Beijersbergen, M.W.; Spreeuw, R.J.C.; Woerdman, J.P.; Barnett, S.M.; Padgett, M.J. Orbital angular momentum of light and the transformation of Laguerre-Gaussian laser modes. Optical. Angular. Momentum. 2004, 45, 8185-8189. [CrossRef]

2. Krenn, M.; Fickler, R.; Fink, M.; Handsteiner, J.; Malik, M.; Scheidl, T.; Ursin, R.; Zeilinger, A. Communication with spatially modulated light through turbulent air across Vienna. New J. Phys. 2014, 16. [CrossRef]

3. Paterson, C. Atmospheric Turbulence and Orbital Angular Momentum of Single Photons for Optical Communication. Phys. Rev. Lett. 2005, 94, 153901. [CrossRef]

4. Krenn, M.; Handsteinera, J.; Fink, M.; Fickler, F.; Ursin, R.; Malik, M.; Zeilinge, A. Twisted light transmission over 143 km. PNAS 2016, 113, 13648-13653. [CrossRef]

5. Qasem, Z.A.H.; Leftah, H.A.; Sun, H.; Qi, J.; Esmaiel, H. X-Transform Time-Domain Synchronous IM-OFDM-SS for Underwater Acoustic Communication. IEEE Syst. J. 2021, 99, 1-12. [CrossRef]

6. Qasem, A.H.; JWang, J.; Kuai, X.; Sun, H.; Esmaiel, H. Enabling Unique Word OFDM for Underwater Acoustic Communication; IEEE Wireless Communications Letters: Xiamen, China, 2021. [CrossRef]

7. Qasem, A.H.; Leftah, H.A.; Sun, H.; Qi, J.; Wang, J.; Esmaiel, H. Deep learning-based code indexed modulation for autonomous underwater vehicles systems. Veh. Commun. 2021, 28, 100314.

8. Cheng, M.; Guo, L.; Li, J.; Huang, Q.; Cheng, Q.; Zhang, D. Propagation of an optical vortex carried by a partially coherent Laguerre-Gaussian beam in turbulent ocean. Appl. Opt. 2016, 55, 4642-4648. [CrossRef]

9. Li, Y.; Cui, Z.; Han, Y.; Hui, Y. Channel capacity of orbital-angular-momentum-based wireless communication systems with partially coherent elegant Laguerre-Gaussian beams in oceanic turbulence. J. Opt. Soc. Am. A 2019, 36, 471-477. [CrossRef]

10. Zhang, W.; Wang, L.; Wang, W.; Zhao, S. Propagation property of Laguerre-Gaussian beams carrying fractional orbital angular momentum in an underwater channel. OSA Contin. 2019, 2, 3281-3287. [CrossRef]

11. Cheng, M.; Guo, L.; Li, J.; Zhang, Y. Channel Capacity of the OAM-Based Free-Space Optical Communication Links with Bessel-Gauss Beams in Turbulent Ocean. IEEE Photon. J. 2016, 8, 1-11. [CrossRef]

12. Chen, M.; Zhang, Y. Effects of anisotropic oceanic turbulence on the propagation of the OAM mode of a partially coherent modified Bessel correlated vortex beam. Waves Random. Complex. Media. 2019, 29, 694-705. [CrossRef]

13. Deng, S.; Yang, D.Y.; Zhang, Y. Capacity of communication link with carrier of vortex localized wave in absorptive turbulent seawater. Waves Random. Complex. Media. 2020, 1-14. [CrossRef]

14. Wang, X.; Yang, Z.; Zhao, S. Influence of oceanic turbulence on propagation of Airy vortex beam carrying orbital angular momentum. Optik 2019, 176, 49-55. [CrossRef]

15. Yu, L.; Zhang, Y. Analysis of modal crosstalk for communication in turbulent ocean using Lommel-Gaussian beam. Opt. Express 2017, 25, 22565-22574. [CrossRef]

16. Hu, Z.; Liu, H.; Xia, J.; He, A.; Li, H.; Du, Z.; Chen, T.; Li, Z.; Lü, Y. Propagation characteristics of the perfect vortex beam in anisotropic oceanic turbulence. Appl. Opt. 2020, 59, 9956-9962. [CrossRef]

17. Liang, Q.; Yang, D.; Zhang, Y.; Zheng, Y.; Hu, L. Probability of orbital angular momentum modes carried by a finite energy frozen wave in turbulent seawater. OSA Contin. 2020, 3, 2429. [CrossRef]

18. Wang, X.; Wang, L.; Zhao, S. Research on Hypergeometric-Gaussian Vortex Beam Propagating under Oceanic Turbulence by Theoretical Derivation and Numerical Simulation. J. Mar. Sci. Eng. 2021, 9, 442. [CrossRef]

19. Zhang, Y.; Zheng, Y.; Yan, Q.; Yu, L. Average capacity of an absorptive turbulent ocean channel with diffraction-and attenuationresistant beam carriers. Opt. Commun. 2021, 499, 127291. [CrossRef]

20. Deng, S.; Yang, D.; Hu, L.; Zhang, Y. Transmittance of Diffraction- and Attenuation-Resistant Vortex Frozen Beams in Oceanic Turbulence; PIERS Fall: Rome, Italy, 2019; pp. 2528-2621. 
21. Deng, S.; Zhu, Y.; Zhang, Y. Received Probability of Vortex Modes Carried by Localized Wave of Bessel-Gaussian Amplitude Envelope in Turbulent Seawater. J. Mar. Sci. Eng. 2019, 7, 203. [CrossRef]

22. Yang, D.; Zhang, Y.; Shi, H. Capacity of turbulent ocean links with carrier Bessel-Gaussian localized vortex waves. Appl. Opt. 2019, 58, 9484-9490. [CrossRef]

23. Zheng, Y.; Yang, D.; Qin, S.; Zhang, Y. Received probability of orbital-angular-momentum modes carried by diffraction- and attenuation resistant beams in weak turbulent oceans. J. Mar. Sci. Eng. 2020, 8, 701. [CrossRef]

24. Li, Y.; Zhang, Y.; Zhu, Y. Oceanic spectrum of unstable stratification turbulence with outer scale and scintillation index of Gaussian-beam wave. Opt. Express 2019, 27, 7656-7672. [CrossRef]

25. Andrews, L.C.; Phillips, R.L. Laser Beam Propagation through Random Media; SPIE Optical Engineering Press: Bellingham, WA, USA, 1998; pp. 47-50.

26. Kotlyar, V.V.; Kovalev, A.A.; Porfirev, A.P. Vortex Laser Beams; Taylor \& Francis Group; CRC Press: Boca Raton, FL, USA, 2019.

27. Bazhenov, V.; Vasnetsov, M.V.; Soskin, M.S. Laser-beam with screw dislocations in the wavefrom. JETP Lett. 1990, 52, 429-431.

28. Yousif, B.B.; Elsayed, E.E. Performance enhancement of an orbital-angular-momentum-multiplexed free-space optical link under atmospheric turbulence effects using apatial-mode multiplexing and hybrid diversity based on adaptive MIMO equalization. IEEE Acess 2020, 7, 84401-84412. [CrossRef]

29. Anguita, J.A.; Neifeld, M.A.; Vasic, B.V. Turbulence-induced channel crosstalk in an orbital angular momentum-multiplexed free-space optical link. Appl. Opt. 2008, 47, 2414-2429. [CrossRef]

30. Tyler, A.G.; Boyd, R.W. Influence of atmospheric turbulence on the propagation of quantum states of light carrying orbital angular momentum. Opt. Lett. 2009, 34, 142-144. [CrossRef]

31. Keskin, A.; Baykal, Y. Effects of underwater turbulence on average transmittance of cos-Gaussian and cosh-Gaussian optical beams. Waves Random. Complex. Media. 2020, 1-12. [CrossRef]

32. Gradshteyn, I.S.; Ryzhik, I.M. Table of Integrals, Series, and Products; Academic Press: Cambridge, MA, USA, 2007.

33. Korotkova, O. Random Light Beams Theory and Applications; CRC Press: Boca Raton, FL, USA, 2014. 